\title{
Efeito da taxa de secreção de néctar sobre a polinização e a produção de sementes em flores de Passiflora speciosa Gardn. (Passifloraceae) no Pantanal
}

\author{
JOSÉ MILTON LONGO1,3 e ERICH FISCHER ${ }^{2}$
}

(recebido: 28 de abril de 2005; aceito: 10 de agosto de 2006)

\begin{abstract}
Effect of nectar secretion rate on pollination and seed production by Passiflora speciosa Gardn. flowers (Passifloraceae) in the Pantanal). We verified the effect of nectar secretion rate (NSR) on the pollinator frequency and the number of seeds per Passiflora speciosa Gardn. flowers in the Pantanal Wetlands. The plant was pollinated by hummingbirds and presented self-incompatibility. The NSR varied as a function of flower diameter, and number of hummingbird visits was a function of flower diameter. The number of seeds per flower of $P$. speciosa increased with the number of pollinator visits. The frequency of nectar thieves had no significant effect on seed set, but total number of hummingbird visits was negatively correlated with the total number of visits by nectar and/or pollen thieves. The overall results indicate that flower size affects NSR, which could determine the number of pollinator visits and the success of seed set per Passiflora speciosa flowers.
\end{abstract}

Key words - flower size, foraging, hummingbird, nectar secretion, seed set

RESUMO - (Efeito da taxa de secreção de néctar sobre a polinização e a produção de sementes em flores de Passiflora speciosa Gardn. (Passifloraceae) no Pantanal). Foi determinado o efeito da taxa de secreção de néctar (TSN) sobre a freqüência de visitas dos polinizadores e o número de sementes por flor de Passiflora speciosa Gardn. no Pantanal. A planta foi polinizada por beija-flores e apresentou auto-incompatibilidade. A TSN variou em função do diâmetro da flor, e o número de visitas dos beija-flores foi função do diâmetro da flor. O número de sementes por flor de $P$. speciosa foi maior com o aumento do número de visitas dos polinizadores. A freqüência de pilhadores de néctar não apresentou efeito sobre a produção de sementes, mas o número total de visitas dos beija-flores foi negativamente correlacionado com o número de visitas dos pilhadores de néctar e/ou pólen. Os resultados indicam que o tamanho da flor afeta a TSN, que por sua vez pode determinar o número de visitas dos polinizadores e o sucesso da produção de sementes em flores de $P$. speciosa.

Palavras-chave - beija-flores, forrageamento, produção de sementes, secreção de néctar, tamanho da flor

\section{Introdução}

Passifloraceae compreende espécies arbóreas, arbustivas, lianas e herbáceas. Reúne aproximadamente 600 espécies, das quais 400 pertencem ao gênero Passiflora L., o qual mais de $25 \%$ de suas espécies ocorrem no Brasil e menos de $10 \%$ delas fora das Américas (Killip 1938). Estudos sobre a biologia floral e a polinização de espécies de Passiflora indicam a predominância da melitofilia e ornitofilia sobre a quiropterofilia (Sazima \& Sazima 1978, Koschnitzke \& Sazima 1997, Varassin et al. 2001, Fischer et al. 2004). Passiflora speciosa Gardn. é liana cujas flores são auto-incompatíveis e polinizadas por beija-flores na Mata Atlântica (Varassin et al. 2001).

\footnotetext{
1. Universidade Federal de Mato Grosso do Sul, Centro de Ciências Biológicas e da Saúde, Programa de Pós-Graduação em Ecologia e Conservação, 79070-900 Campo Grande, MS, Brasil.

2. Universidade Federal de Mato Grosso do Sul, Centro de Ciências Biológicas e da Saúde, Departamento de Biologia, 79070-900 Campo Grande, MS, Brasil.

3. Autor para correspondência: jmlongo@nin.ufms.br
}

Espécies de Passiflora polinizadas por beija-flores oferecem néctar como única recompensa (e.g. Varassin et al. 2001, Fischer \& Leal 2006). A secreção de néctar pode variar em função do local, do horário, da idade e do tamanho da flor e/ou em função de condições ambientais externas (Zimmerman \& Pyke 1988, Sazima et al. 1994, Vicentini \& Fischer 1999). A disponibilidade e a distribuição do néctar entre flores pode determinar o comportamento de polinizadores com respeito à freqüência de visitas, ao número de flores visitadas e ao tempo de duração da visita (Rathcke 1992). Fischer \& Leal (2006) reportam que o tamanho das flores de Passiflora coccinea Aubl. determina a variação da taxa de secreção de néctar e a freqüência de visitas dos polinizadores.

Foram descritos a biologia floral, o sistema reprodutivo e os polinizadores e pilhadores de P. speciosa no Pantanal do Miranda-Abobral, Mato Grosso do Sul. Foi avaliado o efeito da taxa de secreção de néctar (TSN) sobre o sucesso reprodutivo de flores individuais desta espécie através do comportamento dos visitantes florais. Foram testadas as seguintes hipóteses: (1) que a TSN e a freqüência de visitas dos polinizadores 
são determinadas pelo tamanho da flor (diâmetro do hipanto); (2) que a freqüência de visitas dos polinizadores e pilhadores afeta o número de sementes produzidas por flor de P. speciosa.

\section{Material e métodos}

O estudo foi desenvolvido em quatro agrupamentos de indivíduos de Passiflora speciosa Gardn., distantes pelo menos $1 \mathrm{~km}$ entre si, em vegetação ciliar do Rio Miranda, Pantanal do Miranda-Abobral, Mato Grosso do Sul (19³4' S e $\left.57^{\circ} 01^{\prime} \mathrm{W}\right)$. A região apresenta período chuvoso de outubro a março, com pluviosidade média mensal de $200 \mathrm{~mm}$, e período seco de abril a setembro ( $30 \mathrm{~mm}$ mensais), com temperaturas variando de $23{ }^{\circ} \mathrm{C}$ a $34{ }^{\circ} \mathrm{C}$ e de $8{ }^{\circ} \mathrm{Ca} 23^{\circ} \mathrm{C}$, respectivamente (Tarifa 1986).

A biologia floral e a polinização de $P$. speciosa foram estudadas nos meses de janeiro a março de 2000 e de fevereiro a abril de 2001. O horário de antese, a modificação na posição dos elementos florais, a apresentação do néctar e a receptividade do estigma foram registrados durante o período de antese das flores. A receptividade do estigma foi verificada através da presença de exsudato na superfície estigmática e pela atividade catalítica com $\mathrm{H}_{2} \mathrm{O}_{2}$ ao longo do período de antese (Dafni 1992). A altura do androginóforo foi tomada a partir de flores conservadas em etanol 70\%. O material testemunho foi depositado no herbário CGMS/UFMS, Campo Grande, Mato Grosso do Sul.

Para verificar se a taxa de secreção de néctar varia em função do diâmetro da câmara nectarífera, foram ensacadas 11 flores em pré-antese. Essas flores foram então monitoradas ao longo de um mesmo dia. Para cada flor foram medidas as concentrações de açúcares e o volume de néctar em intervalos constantes (90 min), em quatro turnos de amostragem: às $12 \mathrm{~h} 30,14 \mathrm{~h} 00,15 \mathrm{~h} 30 \mathrm{e} 17 \mathrm{~h} 00$. Após cada turno de amostragem, o néctar foi descartado e as flores foram novamente ensacadas para impedir o acesso de visitantes. Em cada turno, essas flores foram amostradas seguindo a mesma seqüência, de modo a manter o mesmo intervalo de tempo entre as medidas em cada flor. $O$ volume de néctar foi tomado com microcapilar graduado (precisão $\pm 0,2 \mu \mathrm{L}$ ) e a concentração de açúcares no néctar com refratômetro portátil $\mathrm{Atago}^{\circledR}(0 \%$ - 32\%). Após a última amostragem de néctar, as flores foram coletadas, sendo medido o diâmetro da base do hipanto com auxílio de paquímetro (precisão $0,1 \mathrm{~mm}$ ). Para testar o efeito do diâmetro externo do hipanto (DIA) sobre a variação da taxa de secreção de néctar (TSN), foram feitas regressões de TSN em função de DIA para cada intervalo de tempo medido, bem como utilizando a produção total entre $11 \mathrm{~h} 00 \mathrm{e} 17 \mathrm{~h} 00$.

O sistema reprodutivo de P. speciosa foi estudado através de experimentos controlados (Dafni 1992). Foram escolhidos 20 indivíduos diferentes e marcado um ramo reprodutivo por indivíduo, com um único botão em pré-antese em cada ramo. Dez botões de indivíduos diferentes foram ensacados e utilizados nos experimentos de autopolinização manual e dez botões não foram ensacados, para verificar a formação de frutos em condições naturais.

A identificação, o período de atividade e o comportamento dos animais visitantes às flores de $P$. speciosa foram registrados através de coletas, de observações diretas, de fotografias e de filmagens durante todo o período de antese das flores. Os insetos visitantes foram capturados e conservados em etanol 70\%. Para os beija-flores visitantes, foi registrado o número de visitas a flores individuais. Ao final da antese, as flores foram marcadas e os frutos foram coletados após 20 dias para contagem do número de sementes presentes. Foram marcadas flores visitadas por diferentes espécies de beija-flores, bem como flores visitadas exclusivamente por Phaethornis eurynome (Lesson) e flores visitadas apenas por Eupetomena macroura (Gmelin). Foram feitas regressões para testar se a freqüência de visitas dos polinizadores é determinada pelo diâmetro da flor, assim como se o número de sementes produzidas por flor é determinado pelo número de visitas de polinizadores ou de pilhadores. Foram feitas análises de correlação entre o número de visitas de polinizadores e pilhadores, para verificar relação entre esses dois grupos de visitantes florais. Foram utilizados testes de Chi-quadrado $\left(\chi^{2}\right)$ para verificar diferenças da proporção de frutos formados entre as flores visitadas pelos beija-flores P. eurynome e E. macroura, e análise de variância para determinar diferenças do número de sementes produzidas por flores visitadas por estas duas espécies de beija-flores. As médias apresentadas são acompanhadas do erro padrão.

\section{Resultados}

As flores de $P$. speciosa Gardn. são inodoras, solitárias e eretas; possuem zigomorfia sutil, devido ao maior espaçamento entre dois dos três filetes, e ficam destacadas da folhagem pelo longo pedúnculo e pedicelo $(\sim 10 \mathrm{~cm})$. A câmara nectarífera está encerrada no hipanto, subdividida em subcâmaras protegidas por um opérculo membranáceo e rígido na parte superior. $\mathrm{O}$ androginóforo mede $29,1 \pm 0,16 \mathrm{~mm}(n=30)$ de altura e o diâmetro do hipanto $13,3 \pm 0,22 \mathrm{~mm}(n=11)$. As sépalas e pétalas apresentam aproximadamente $37 \mathrm{e}$ $36 \mathrm{~mm}$ de comprimento, respectivamente. As sépalas e pétalas são vermelhas, assim como os filetes, estiletes e as extremidades das fímbrias. As fímbrias são carnosas e rígidas ( $\sim 15 \mathrm{~mm}$ de comprimento), formando uma barreira ao acesso à câmara nectarífera.

No Pantanal do Miranda-Abobral, P. speciosa floresceu durante todo o período de estudo. Os ramos reprodutivos ocorreram entre 0,1 e $12 \mathrm{~m}$ de altura. A abertura das flores iniciava entre $8 \mathrm{~h} 15 \mathrm{e} 9 \mathrm{~h} 00$. As flores foram protândricas, uma vez que no momento da 
abertura os estigmas não estavam receptivos, porém havia pólen disponível. Os estigmas estavam receptivos a partir de $9 \mathrm{~h} 30$, permanecendo assim até às $18 \mathrm{~h} 00 \mathrm{ou}$ 19h00. Cerca de duas horas após a abertura da flor, os estiletes iniciavam movimento para baixo, até a superfície estigmática atingir o mesmo nível das anteras, entre $11 \mathrm{~h} 00$ e $11 \mathrm{~h} 30$. Por volta de $15 \mathrm{~h} 30$, os estigmas retornaram à posição anterior, ocupada no momento da abertura da flor. As brácteas, sépalas e pétalas também retornaram à posição adotada antes da abertura, fechando a flor entre $18 \mathrm{~h} 00$ e $19 \mathrm{~h} 00$.

As flores começaram a secretar néctar aproximadamente uma hora após a sua abertura e cessaram a produção uma hora antes de fecharem. $\mathrm{O}$ volume médio de néctar acumulado entre os intervalos de amostragem de $90 \mathrm{~min}$ foi de $6,1 \pm 0,26 \mu \mathrm{L}(n=11)$ e a concentração média do néctar foi de $21,7 \pm 0,28 \%$ $(n=11)$ (figura 1). O volume não foi significativamente correlacionado $(r=0,09 ; P=0,79 ; n=11)$ com a concentração do néctar. A taxa média de secreção de néctar (TSN) foi de $0,068 \pm 0,003 \mu \mathrm{L} \mathrm{min}^{-1}(n=11)$ e o diâmetro do hipanto (DIA), foi em média de 13,3 \pm $0,2 \mathrm{~mm}(n=11)$. Considerando todo volume produzido entre $11 \mathrm{~h} 00$ e $17 \mathrm{~h} 00$, a TSN variou em função do DIA $\left(\mathrm{TSN}=-0,101+0,013\right.$ DIA; $\left.P<0,0001 ; r^{2}=0,97\right)$ assim como ocorreu para análises parciais para cada classe de horário (tabela 1; figura 2). Os valores de $r^{2}$ em diferentes intervalos de amostragem (tabela 1), indicaram que 66\%-76\% da variação da TSN é determinada pela variação do diâmetro do hipanto (DIA). Somente no último intervalo de amostragem (15h31-17h00) não houve relação significativa da $\mathrm{TSN}=\mathrm{f}$ (DIA).

A proporção de frutos formados a partir de flores autopolinizadas manualmente $(0 / 10)$ foi significativamente menor $(P<0,03$; Teste Exato de Fisher) que a proporção de frutos formados a partir de flores visitadas em condições naturais (6/10).

As flores de $P$. speciosa foram visitadas por quatro espécies de beija-flores, Eupetomena macroura (Gmelin), Phaethornis eurynome (Lesson), Hylocharis chrysura (Shaw) e Polytmus guainumbi (Linné). Durante as visitas, essas espécies contataram os órgãos reprodutivos das flores com a parte anterior da cabeça. Eupetomena macroura foi o beija-flor mais freqüente em flores de $P$. speciosa no local de estudo, tendo visitado 50 das 71 flores focais (c. de 70\%). A primeira visita de E. macroura foi registrada logo após a abertura da flor, às 8 h20 e a última visita às 18 h40. Após visitar algumas flores, pousava em ramos nas proximidades, geralmente em galhos mais altos. Os indivíduos de E. macroura defenderam territórios que incluíam flores de P. speciosa e de plantas vizinhas também floridas, Helicteres guazumaefolia H.B.K. (Sterculiaceae) e Inga vera (DC) T.D. Penn (Fabaceae). A média de visitas de E. macroura às flores focais de $P$. speciosa, foi de 3,9 \pm 0,52; com um total de 199 visitas em 50 flores (tabela 2). Phaethornis eurynome visitou 34 das 71 flores focais de $P$. speciosa (c. de 48\%), a partir das $8 \mathrm{~h} 20$ até o fechamento das flores, às $19 \mathrm{~h} 00$. Este beijaflor visitava seqüencialmente de oito a dez flores, voando em seguida para fora da mancha de P. speciosa. A média de visitas por flor foi de $3,9 \pm 0,44$, com 131 visitas em 34 flores (tabela 2). Hylocharis chrysura e Polytmus guainumbi foram visitantes menos freqüentes das flores de P. speciosa, tendo visitado dez e quatro flores, respectivamente. Entretanto, não ocorreram como visitantes exclusivos em nenhuma dessas flores. Visitaram principalmente flores localizadas em ramos mais baixos, aproximando-se das flores em vôos rápidos e realizando visitas breves (1-2 s). O número médio de visitas por flor foi de 2,1 $\pm 0,3$ para $H$. chrysura e de $1,3 \pm 0,3$ para $P$. guainumbi.

O total de visitas dos beija-flores durante o período

Tabela 1. Regressões da taxa de secreção de néctar (TSN) como função do diâmetro da base (DIA) das flores de Passiflora speciosa Gardn. (Passifloraceae) em diferentes intervalos de amostragem, no Pantanal do Miranda-Abobral.

Table 1. Regressions of nectar secretion rate (TSN) as a function of Passiflora speciosa Gardn. (Passifloraceae) flower base diameter (DIA) in different sample intervals, in the "Pantanal do Miranda-Abobral".

\begin{tabular}{lcccc}
\hline Intervalo & Função estimada & $n$ & $P$ & $r^{2}$ \\
\hline $11 \mathrm{~h} 00-12 \mathrm{~h} 30$ & TSN $=-0,061+0,007$ DIA & 11 & 0,0023 & 0,66 \\
$12 \mathrm{~h} 31-14 \mathrm{~h} 00$ & TSN $=-0,099+0,013$ DIA & 11 & 0,0008 & 0,73 \\
$14 \mathrm{~h} 01-15 \mathrm{~h} 30$ & TSN $=-0,226+0,026$ DIA & 11 & 0,0004 & 0,77 \\
$15 \mathrm{~h} 31-17 \mathrm{~h} 00$ & Não significativa & 11 & 0,1773 & 0,19 \\
Secreção total & TSN =-0,101+0,013 DIA & 11 & 0,0001 & 0,97 \\
\hline
\end{tabular}


de antese variou significativamente em função do diâmetro externo do hipanto das flores $\left(r^{2}=0,16\right.$; $P<0,001 ; n=70$ ) (figura 3). O total de visitas dos beijaflores em flores individuais foi negativamente correlacionado $(r=-0,25 ; P<0,02 ; n=88)$ ao total de visitas de pilhadores. A média de sementes por fruto foi de $118 \pm 97,1(n=41)$. O número de sementes produzidas por flor aumentou assintoticamente com o aumento do número total de visitas dos polinizadores $\left(r^{2}=0,31\right.$; $P<0,001 ; n=41)$ (figura 4), entretanto, não houve efeito da freqüência de pilhadores sobre a produção de sementes $(P=0,15 ; n=41)$. Das 79 flores focais estudadas, 71 receberam visitas de beija-flores e 41 formaram frutos $(57,7 \%)$. A proporção de frutos formados (19/41), dentre as flores visitadas exclusivamente por $E$. macroura, não foi significativamente diferente $\left(\chi^{2}=0,61 ; g l=1 ; P=0,43\right)$
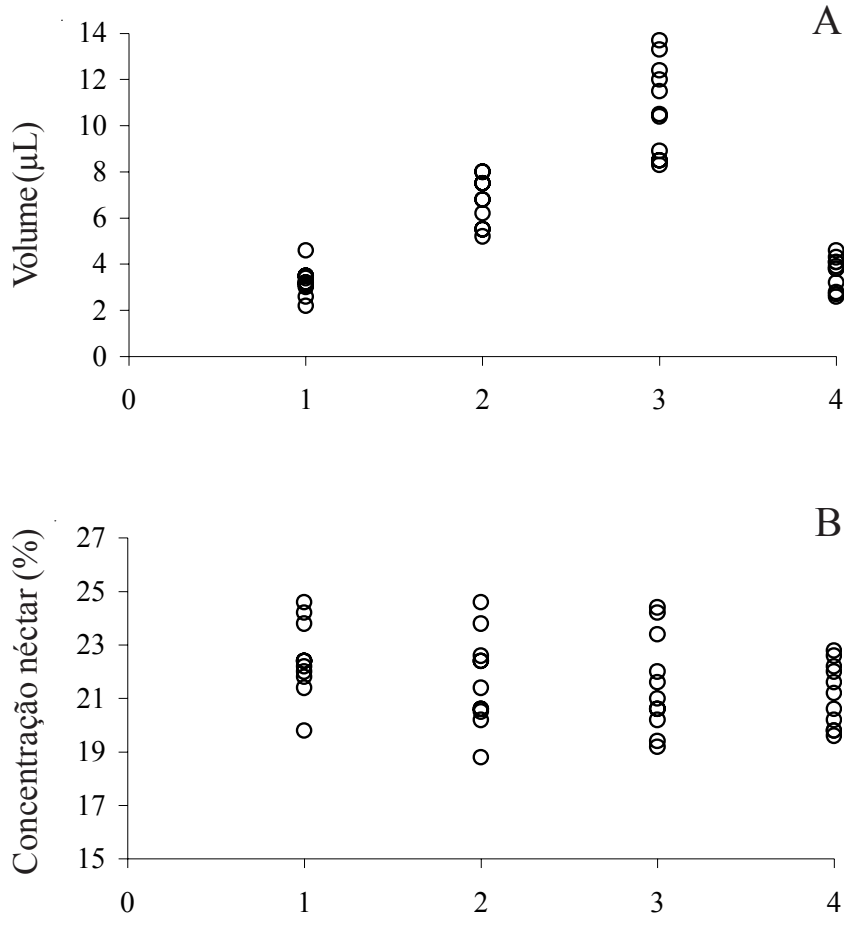

Classes de horário

Figura 1. Volume $(\mu \mathrm{L})$ de néctar acumulado durante $90 \mathrm{~min}$ (A) e concentração do néctar (\%) (B) em flores de Passiflora speciosa Gardn. (Passifloraceae) em diferentes classes de horário, no Pantanal do Miranda-Abobral. Classes de horário: $1=11 \mathrm{~h} 00-12 \mathrm{~h} 30 ; 2=12 \mathrm{~h} 31-14 \mathrm{~h} 00 ; 3=14 \mathrm{~h} 01-15 \mathrm{~h} 30 ; 4=15 \mathrm{~h} 31$ $17 \mathrm{~h} 00$.

Figure 1. Nectar volume ( $\mu \mathrm{L})$ accumulated during $90 \mathrm{~min}(\mathrm{~A})$ and nectar concentration (\%) (B) in Passiflora speciosa Gardn. (Passifloraceae) in different time classes in the "Pantanal do Miranda-Abobral". Time classes: $1=11 \mathrm{~h}-12: 30$; $2=12: 31-14 \mathrm{~h} ; 3=14: 01-15: 30 ; 4=15: 31-17 \mathrm{~h}$.

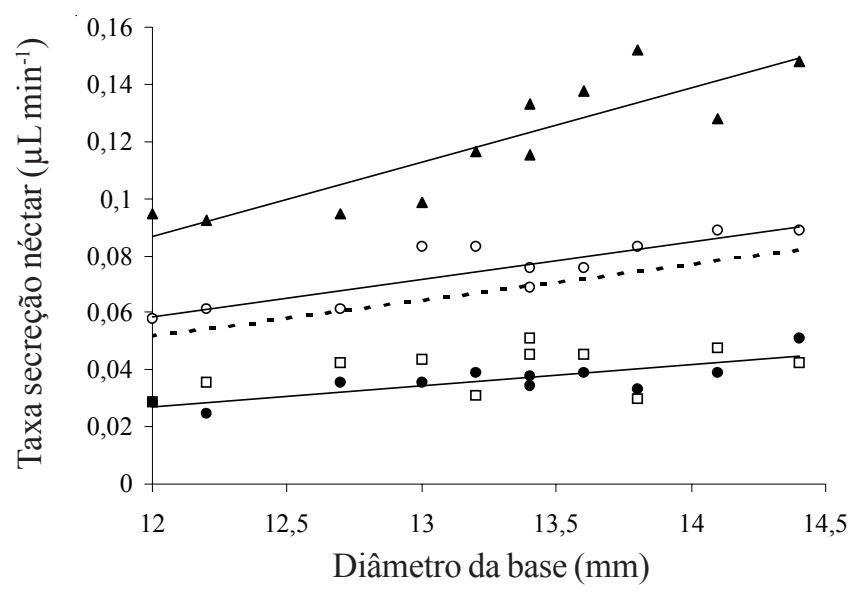

Figura 2. Taxa de secreção de néctar $\left(\mu \mathrm{L} \min ^{-1}\right)$ em função do diâmetro $(\mathrm{mm})$ da base das flores de Passiflora speciosa Gardn. (Passifloraceae), em diferentes classes de horário no Pantanal do Miranda-Abobral. Linhas contínuas correspondem às funções estimadas para cada intervalo de amostragem $(\bullet=11 \mathrm{~h} 00-12 \mathrm{~h} 30 ; \mathrm{O}=12 \mathrm{~h} 31-14 \mathrm{~h} 00 ; \boldsymbol{\Delta}=14 \mathrm{~h} 01$ $15 \mathrm{~h} 30 ; \square=15 \mathrm{~h} 31-17 \mathrm{~h} 00$ ) e a linha pontilhada representa a função estimada tomando a produção de todos os intervalos. No intervalo entre $15 \mathrm{~h} 31$ e $17 \mathrm{~h} 00$ a função não foi significativa e a linha de tendência não está incluída. Veja funções estimadas na tabela 1 .

Figure 2. Nectar secretion rate $\left(\mu \mathrm{L} \min ^{-1}\right)$ as a function of Passiflora speciosa Gardn. (Passifloraceae) flower base diameter $(\mathrm{mm})$, in different time classes in the "Pantanal do Miranda-Abobral". Continuous lines indicate functions estimated for each time class $(\bullet=11 \mathrm{~h}-12: 30 ; \bigcirc=12: 31-14 \mathrm{~h}$;

$\boldsymbol{\Delta}=14: 01-15: 30 ; \square=15: 31-17 \mathrm{~h}$ ), and dotted line indicates the estimated function taking the production of all time classes. In the 15:31-17h time interval, the estimated function was not significant so the line of tendency is not presented. See the estimated functions in table 1 .

da proporção de frutos formados dentre as flores visitadas apenas por P. eurynome (9/13). Entretanto, as flores visitadas por $P$. eurynome produziram significativamente $\left(\mathrm{F}_{1-26}=4,86 ; P<0,05\right)$ mais sementes $(213,1 \pm 29,62 ; n=9)$ do que flores visitadas exclusivamente por $E$. macroura $(145,5 \pm 15,86 ; n=19)$.

Os insetos registrados como visitantes das flores de $P$. speciosa foram pilhadores de néctar e/ou pólen (tabela 2). Dentre os pilhadores, as abelhas Trigona spinipes (Fabricius), Xylocopa sp. e Apis mellifera (Linnaeus), foram as mais freqüentes. Trigona spinipes perfurava a base do hipanto para acessar o néctar. Os Passeriformes Saltator atricollis (Vieillot) e Ramphocelus carbo (Pallas) visitaram para consumo de partes florais, pólen e anteras de duas flores abertas de P. speciosa. 


\section{Discussão}

Passiflora speciosa Gardn. apresentou atributos florais que se ajustam aos descritos para a síndrome de ornitofilia. A cor escarlate, a ausência de odor, a distância relativamente grande entre as partes reprodutivas e a fonte de alimento e os atributos do néctar, caracterizam essa planta como ornitófila (Faegri \& van der Pij1 1980,

Tabela 2. Número de flores visitadas, médias ( \pm EP) de visitas por flor, função e recurso floral utilizado por visitantes florais de Passiflora speciosa Gardn. (Passifloraceae) no Pantanal do Miranda-Abobral.

Table 2. Number of flowers visited, mean ( \pm SE) visits per flower, role and floral resource used by floral visitors of Passiflora speciosa Gardn. (Passifloraceae) in the "Pantanal do Miranda-Abobral".

\begin{tabular}{lcclc}
\hline Visitantes florais & Número de flores visitadas & Visitas por flor & Função & Recurso floral \\
\hline TROCHILINAE & & & & \\
$\quad$ Eupetomena macroura & 50 & $3,9 \pm 0,52$ & Polinizador & Néctar \\
$\begin{array}{l}\text { Hylocharis chrysura } \\
\text { Polytmus guainumbi }\end{array}$ & 10 & $2,1 \pm 0,28$ & Polinizador & Néctar \\
PHAETHORNITHINAE & 4 & $1,2 \pm 0,25$ & Polinizador & Néctar \\
$\quad$ Phaethornis eurynome & 34 & $3,8 \pm 0,44$ & Polinizador & Néctar \\
PASSERIFORME & 2 & - & Pilhador & Anteras \\
Ramphocelus carbo & 2 & - & Pilhador & Anteras \\
Saltator atricollis & 19 & - & Pilhador & Pólen/Néctar \\
HYMENOPTERA & 7 & - & Pilhador & Néctar \\
Trigona spinipes & 5 & - & Pilhador & Pólen \\
Xylocopa sp. & 3 & - & Pilhador & Pólen \\
Apis mellifera & & & & \\
Centris sp. & & & & \\
\hline
\end{tabular}

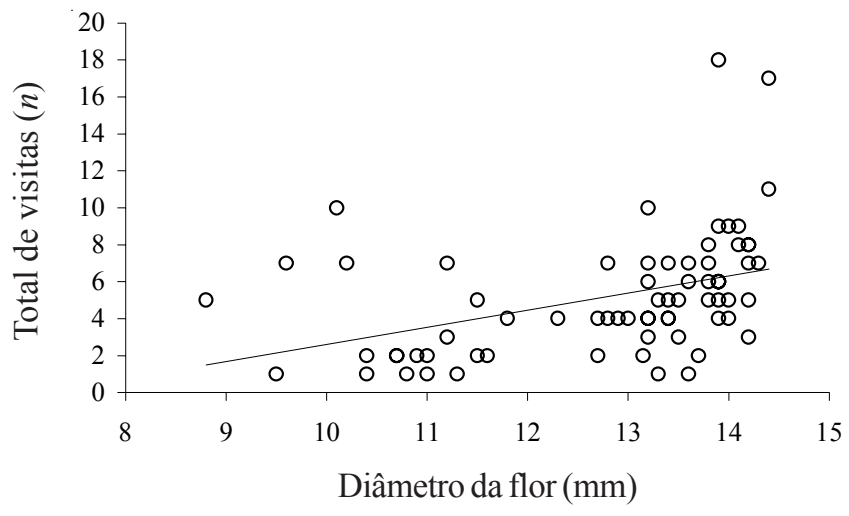

Figura 3. Número total de visitas de beija-flores em função do diâmetro (mm) da base de flores de Passiflora speciosa Gardn. (Passifloraceae) no Pantanal do Miranda-Abobral $\left(Y=7,7+0,9 \mathrm{X} ; r^{2}=0,16 ; P<0,001 ; n=70\right)$.

Figure 3. Total number of hummingbird visits as a function of Passiflora speciosa Gardn. (Passifloraceae) flower base diameter $(\mathrm{mm})$ in the "Pantanal do Miranda-Abobral" $(Y=-7,7$ $\left.+0,9 \mathrm{X} ; r^{2}=0,16 ; P<0,001 ; n=70\right)$.

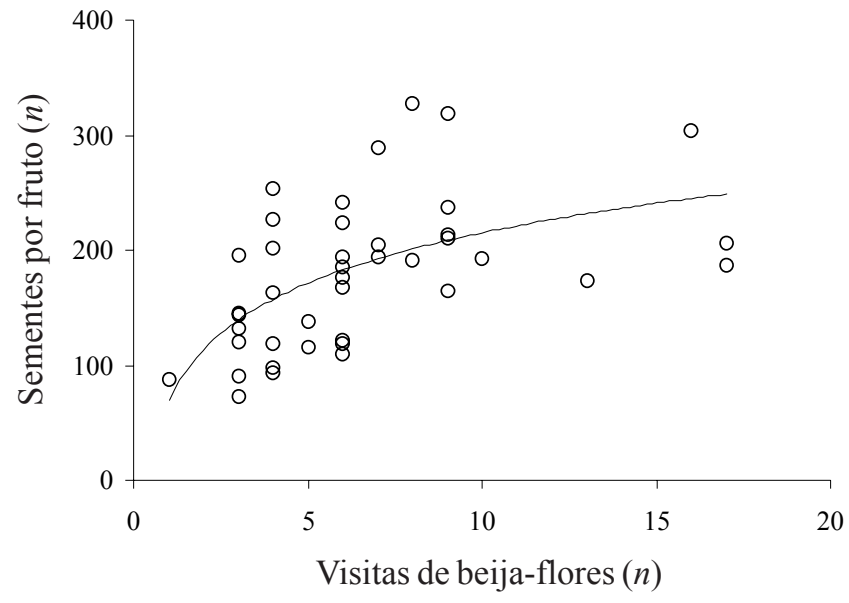

Figura 4. Número de sementes produzidas por flor de Passiflora speciosa Gardn. (Passifloraceae) em função do número de visitas de beija-flores no Pantanal do Miranda$\operatorname{Abobral}\left(Y=69,7+145,4 \log (\mathrm{X}) ; r^{2}=0,31 ; P<0,001 ; n=41\right)$.

Figure 4. Number of seeds produced per Passiflora speciosa Gardn. (Passifloraceae) flower as a function of hummingbird visits $(n)$ in the "Pantanal do Miranda-Abobral" $(Y=69,7+$ $\left.145,4 \log (\mathrm{X}) ; r^{2}=0,31 ; P<0,001 ; n=41\right)$. 
Feinsinger 1983, Varassin et al. 2001). As fímbrias carnosas e rígidas podem impedir o acesso de pilhadores pequenos à câmara nectarífera, entretanto algumas abelhas podem perfurar a base do hipanto, como T. spinipes (Sazima \& Sazima 1989).

No local de estudo, as flores de $P$. speciosa apresentaram néctar com concentração média de açúcares em torno de $22 \%$, valor muito próximo ao reportado para esta espécie na Mata Atlântica e para outras flores ornitófilas (Buzato et al. 2000, Varassin et al. 2001). A concentração média de açúcares no néctar de $P$. speciosa decresceu ao longo do período de antese, ao passo que o volume aumentou durante período de $11 \mathrm{~h} 00$ a $15 \mathrm{~h} 30$. A diminuição do volume de néctar no último intervalo amostrado (15h31 a 17h00) pode estar relacionada ao fim da secreção e/ou à reabsorção do néctar, como registrado para $P$. speciosa na Mata Atlântica (Varassin et al. 2001). Essa baixa produção de néctar registrada entre $15 \mathrm{~h} 31$ e $17 \mathrm{~h} 00$ poderia explicar a ausência de significância da $\mathrm{TSN}=\mathrm{f}$ (DIA) nesta classe de horário.

A relação significativa entre a TSN e o tamanho das flores indica que flores maiores secretaram mais néctar por unidade de tempo. De modo semelhante, Passiflora coccinea Aubl. (Passifloraceae), uma espécie amazônica também polinizada por beija-flores, apresentou variação da TSN em função do diâmetro do hipanto (Fischer \& Leal 2006). Portanto, a utilização do diâmetro do hipanto como variável para estimar a TSN em flores ornitófilas de Passiflora tem se mostrado aplicável. Estudos da relação entre a TSN e o tamanho de flores quiropterófilas mostram a mesma tendência (Fischer 2000). Os experimentos de autopolinização indicaram que $P$. speciosa é autoincompatível, evidenciando a necessidade de pólen alógamo para a formação de frutos. Resultados similares foram obtidos para $P$. speciosa na Mata Atlântica e para outras espécies não ornitófilas de Passiflora (P. mucronata Lam., P. galbana Mast. e P. alata Ait.) (Varassin et al. 2001).

O número de visitas dos beija-flores variou significativamente em função do diâmetro da flor, entretanto a relação apresentou baixo valor para o coeficiente de regressão $\left(r^{2}=0,16\right)$. Este efeito pouco marcante pode ser parcialmente explicado pela inclusão de quatro espécies de beija-flores que apresentam diferentes comportamentos de visita e, portanto, podem responder de modo diverso com respeito à variação do recurso (Feinsinger 1983). Em P. coccinea, visitada exclusivamente por uma espécie de beija-flor, a variação do número de visitas em função do diâmetro das flores apresentou alto valor $\left(r^{2}=0,81\right)$ para o coeficiente de regressão (Fischer \& Leal 2006). Uma vez que a TSN varia em função do DIA, o maior número de visitas em flores maiores pode ser devido à maior disponibilidade de néctar. Isso implica que o comportamento de visita dos beija-flores é sensível às pequenas variações na disponibilidade e distribuição do néctar entre flores coespecíficas, como registrado para flores de P. speciosa, P. coccinea e Ipomopsis aggregata (Pursh) V.E. Grant (Polemoniaceae) (Campbell et al. 1996, Varassin et al. 2001, Fischer \& Leal 2006).

Devido à realização de visitas legítimas e ao contato com os órgãos reprodutivos das flores os beija-flores podem ser considerados os polinizadores de $P$. speciosa no Pantanal do Miranda-Abobral, assim como registrado na Mata Atlântica (Varassin et al. 2001). Com base na freqüência de visitas das espécies de beija-flores registradas, $P$. eurynome e E. macroura são os principais polinizadores desta planta no local de estudo. Os beija-flores Hylocharis chrysura e Polytmus guainumbi podem ser considerados polinizadores secundários devido à irregularidade e baixa freqüência de visitas às flores (Feinsinger 1983, Araujo \& Sazima 2003). Considerando a auto-incompatibilidade de P. speciosa (Varassin et al. 2001, presente estudo), o comportamento de visitas de E. macroura poderia levar à menor produção de sementes por flor, do que o comportamento de $P$. eurynome. O comportamento territorial de $E$. macroura pode favorecer cruzamentos entre flores de um agrupamento de indivíduos, mas reduziria o fluxo de pólen entre flores de diferentes agrupamentos (Feinsinger 1983, Araujo \& Sazima 2003). Adicionalmente, a territorialidade aumentaria a probabilidade de visitas repetidas às mesmas flores e, portanto, autopolinização. Por outro lado, P. eurynome forrageia através de rotas de visitas em plantas espaçadas e com poucas flores abertas diariamente, favorecendo a polinização entre flores de diferentes agrupamentos de P. speciosa. Portanto, o comportamento de visitas de $P$. eurynome promoveria a fecundação cruzada (Feinsinger 1983, Araujo et al. 1994). A diferença do comportamento de visitas entre estas espécies de beija-flores (Araujo \& Sazima 2003) poderia, portanto, explicar a maior produção de sementes em flores visitadas por $P$. eurynome que por E. macroura.

A freqüência dos pilhadores não teve efeito sobre a produção de sementes, indicando que os pilhadores não afetam negativamente a produção de sementes por flor de P. speciosa no local de estudo. Entretanto, a quantidade de visitas dos beija-flores às flores de $P$. speciosa foi negativamente correlacionada ao número de visitas dos pilhadores, apontando possível competição 
entre estes grupos de visitantes pelo néctar de P. speciosa no Pantanal do Miranda-Abobral. Em flores de Passiflora vitifolia H.B.K. a exclusão de Trigona spp. promoveu aumento do número de visitas dos beija-flores, indicando também a competição entre polinizadores e pilhadores (Gill 1988).

O número de sementes produzidas por flor de $P$. speciosa aumentou assintoticamente em função do total de visitas dos polinizadores, sugerindo que a partir de um determinado número de visitas, a quantidade de sementes produzidas tende a estabilizar. Resultado semelhante foi reportado para $P$. vitifolia, cujo número de sementes por flor foi proporcional à quantidade de pólen alógamo depositado nos estigmas (Snow 1982). Adicionalmente, a quantidade de frutos e o número de sementes produzidas por frutos de $P$. edulis Sims tiveram acréscimo com o aumento da intensidade de polinização cruzada (Akamine \& Girolami 1959).

Os resultados deste estudo indicam que o tamanho do hipanto de flores de $P$. speciosa determina $97 \%$ da variação da taxa de secreção de néctar que, por sua vez, deve afetar a freqüência de visitas dos beija-flores, principais polinizadores destas flores (Varassin et al. 2001, Fischer \& Leal 2006). O número de sementes produzidas por flor de $P$. speciosa parece ser parcialmente determinado pela freqüência e comportamento de visitas dos beija-flores. Espécies territoriais, como E. macroura, parecem menos eficientes para a polinização de $P$. speciosa que beijaflores que forrageiam em rotas de visitas, como P. eurynome no Pantanal do Miranda-Abobral.

Agradecimentos - A Andréa Araujo, Maria Rosângela Sigrist e Simone Longo, pela revisão do manuscrito. A Alan Fecchio, Andréa Araujo, Rafael Arruda e Simone Longo, pela ajuda no campo. A Ubirazilda Resende do herbário CGMS/UFMS, pelo auxílio na identificação do material botânico. A Coordenadoria de Estudos do Pantanal (PROPP-UFMS), pelo apoio logístico. À Capes e ao CNPq, pelas bolsas de mestrado ao primeiro autor, e à National Science Foundation pelo financiamento.

\section{Referências bibliográficas}

AKAMINE, E.K. \& GIROLAMI, G. 1959. Pollination and fruit set in the yellow passion fruit. Technical Bulletin $n^{\circ} 39$. Agriculture Experimental Station, Hawaii.

ARAUJO, A.C. \& SAZIMA, M. 2003. The assemblage of flowers visited by hummingbirds in the "capões" of Southern Pantanal, Mato Grosso do Sul, Brazil. Flora 198:427-435.
ARAUJO, A.C., FISCHER, E. \& SAZIMA, M. 1994. Floração seqüencial e polinização de três espécies de Vriesea (Bromeliaceae) na região da Juréia, Sudeste do Brasil. Revista Brasileira de Botânica 17:113-118.

BUZATO, S., SAZIMA, M. \& SAZIMA, I. 2000. Hummingbird pollinated floras at three Atlantic forest sites. Biotropica 32:824-841.

CAMPBELL, D.R., WASER, N.M. \& PRICE, M.V. 1996. Mechanism of hummingbird-mediated selection of flowers width in Ipomopsis aggregata. Ecology 77:1463-1472.

DAFNI, A. 1992. Pollination Ecology - A practical approach. Oxford University Press, Oxford.

FAEGRI, K. \& VAN DER PIJL, L. 1980. The principles of pollination ecology. Pergamon Press, Oxford.

FEINSINGER, P. 1983. Coevolution and pollination. In Coevolution. (D.J. Futuyma \& M. Slatkin, eds.). Sinauer Associates, Sunderland, p.282-310.

FISCHER, E. 2000. Polinização por morcegos Glossophaginae versus Phyllostominae em floresta de terra firme na Amazônia Central. Tese de doutorado, Universidade Estadual de Campinas, Campinas.

FISCHER, E. \& LEAL, I.R. 2006. Effect of nectar secretion rate on pollination success of Passiflora coccinea (Passifloraceae) in the central Amazon. Brazilian Journal of Biology 66:29-41.

FISCHER, E., LEAL, I.R. \& GAONA, J.C. 2004. Polinização de Passiflora nitida (Passifloraceae) e o comportamento territorial de Centris spp. (Hymenoptera: Anthophoridae) em floresta de terra firme na Amazônia Central. In História natural, ecologia e conservação de algumas espécies de plantas e animais da Amazônia (R. Cintra, ed.). Edua / Inpa, Manaus, p.73-77.

GILL, F.B. 1988. Trapline foraging by hermit hummingbirds: competition for an undefended, renewable resource. Ecology 69:1933-1942.

KILLIP, E.P. 1938. The American species of Passifloraceae. Field Museum of Natural History Botanical Series 19:1-613.

KOSCHNITZKE, C. \& SAZIMA, M. 1997. Biologia floral de cinco espécies de Passiflora L. (Passifloraceae) em mata semidecídua. Revista Brasileira de Botânica 20:119-126.

RATHCKE, B.J. 1992. Nectar distribution, pollinator behavior and plant reproductive success. In Effects of resource distribution on animal-plant interactions. (M.D. Hunter, T. Ohgushi \& P.W. Price, eds.). Academic Press, New York, 113-138.

SAZIMA, M. \& SAZIMA, I. 1978. Bat-pollination of the passion flower, Passiflora mucronata, in southeastern Brazil. Biotropica 10:100-109.

SAZIMA, I. \& SAZIMA M. 1989. Mamangavas e irapuás (Hymenoptera, Apoidea): visitas, interações e conseqüências para a polinização do maracujá (Passifloraceae). Revista Brasileira de Biologia 33:109-118. 
SAZIMA, M., SAZIMA, I. \& BUZATO, S. 1994. Nectar by day and night: Siphocampylus sulfureus (Lobeliaceae) pollinated by hummingbirds and bats. Plant Systematics and Evolution 191:237-246.

SNOW, A.A. 1982. Pollination intensity and potential seed set in Passiflora vitifolia. Oecologia 55:231-237.

TARIFA, J.R. 1986. O sistema climático do pantanal: da compreensão do sistema à definição de prioridades de pesquisa climatológica. In Anais do I Simpósio sobre recursos naturais e socioeconômicos do Pantanal. Ministério da Agricultura, Brasília, p.9-28.
VARASSIN, I.G., TRIGO, J.R. \& SAZIMA, M. 2001. The role of production, flower pigments and odour in the pollination of four species of Passiflora (Passifloraceae) in south-eastern Brazil. Botanical Journal of the Linnean Society 136:138-152.

VICENTINI,A. \& FISCHER, E. 1999. Pollination of Moronobea coccinea (Clusiaceae) by the Golden-Winged Parakeet in Central Amazon. Biotropica 31:692-696.

ZIMMERMAN, M. \& PYKE, G.H. 1988. Experimental manipulations of Polemonium foliosissimum: effects on subsequent nectar production, seed production, and growth. Journal of Ecology 76:777-789. 